\title{
ROL DE LAS INSTITUCIONES DE ALTA COMPLEJIDAD EN LA REDUCCIÓN DE LA MORTALIDAD MATERNA Y NEONATAL
}

\section{ROLE OF HIGH COMPLEXITY INSTITUTIONS IN REDUCING MATER- NAL AND NEONATAL MORTALITY}

\author{
Enrique Guevara-Ríos $1,2, a$
}

Los establecimientos de salud de tercer nivel de atención deben tener entre sus políticas institucionales brindar acceso oportuno a los servicios especializados en el área materno perinatal, contribuyendo a la formación de redes de servicios efectivas para la atención, con énfasis en la atención de alta complejidad¹.

El objetivo general es que estos establecimientos dentro del sistema de salud, puedan brindar atención especializada y de calidad en salud sexual y reproductiva con énfasis en la atención de las complicaciones más severas de las gestantes y neonatos, especialmente en los neonatos prematuros ${ }^{2}$.

Para lograr este objetivo general se deben plantear los siguientes objetivos específicos: atención especializada en obstetricia crítica y en medicina materno-fetal; atención especializada en neonatología crítica, desarrollo del potencial humano de la institución y promoción de investigaciones que puedan contribuir a mejorar la salud de las gestantes y neonatos en el país ${ }^{3}$.

Para alcanzar una atención especializada en obstetricia crítica y en medicina materno-fetal se requiere estandarización de los procesos de atención mediante guías de atención basadas en evidencia científica de las principales patologías maternas que llevan a la mortalidad materna y a la morbilidad materna extremadamente grave como son la preeclampsia, la sepsis obstétrica y no obstétrica, la hemorragia post parto, el aborto, y el acretismo placentario ${ }^{4,5}$. Asimismo, se requiere estandarizar los procedimientos para la cirugía fetal intrauterina y la ecografía obstétrica. Se requiere también implementar salas de shock trauma para gestantes en el Servicio de Emergencia, manejo multidisciplinario de las claves roja, azul y amarilla, implementar y fortalecer Unidades de Cuidados Intensivos Materna (UCIM) con equipos de última generación y con recursos humanos capacitados en obstetricia crítica. Y en estos tiempos de pandemia por COVID-19 se deben implementar UCIM para gestantes con COVID-19 con compromiso respiratorio severo. Es importante un adecuado sistema de referencias y contrarreferencias, así como el uso de la telemedicina para el manejo inicial de las emergencias obstétricas.

Para lograr una atención especializada en neonatología crítica se requiere estandarizar las guías de práctica clínica de Neonatología, que consideren el manejo del recién nacido extremadamente prematuro, sepsis neonatal, asfixia neonatal entre otras patologías relacionadas con la mortalidad neonatal. Se debe buscar el fortalecimiento de las Unidades de Cuidados Intensivos e Intermedios Neonatales con incubadoras, ventiladores mecánicos neonatales, monitores multiparámetros, equipos de hipotermia, equipos de electroencefalografía, ecógrafos, asi como de recursos humanos capacitados para el manejo en estas unidades. La implementación de bancos de leche humana y la promoción de la lactancia materna deben ser prioridades institucionales.

Existen estrategias transversales que permiten alcanzar estos dos objetivos específicos: Rondas de Seguridad, informe de eventos adversos durante la atención, supervisión del desempeño de los equipos de guardia y llevar a cabo el proceso anual de autoevaluación de los estándares de calidad de atención materno-neonatales.

El desarrollo del potencial humano se logra a través de educación continua del personal de salud, a través de las capacitaciones organizadas para la mejora de la calidad de la atención como son los cursos de Gestión Hospitalaria, cursos de formación de evaluadores internos, y cursos excelencia en el trato al Usuario. Tambien a través de las capacitaciones organizadas para la implementación del uso de la Historia Clínica Perinatal SIP/PLUS, que incorpora el análisis de

Instituto Nacional Materno Perinatal. Lima, Perú.

Departamento de Ginecología y Obstetricia. Universidad Nacional Mayor de San Marcos. Lima,Perú.

Médico Ginecólogo-Obstetra. Director del Instituto Nacional Materno Perinatal. Profesor Ordinario de la Facultad de Medicina, Universidad Nacional Mayor de San Marcos Lima-Perú. Coordinador de Asistencia Técnica del Instituto de Salud Popular.

(iD) Código ORCID: https://orcid.org/0000-0002-6962-2639, Enrique Guevara Rios

Citar como: Guevara Ríos E. Rol de las instituciones de alta complejidad en la reducción de la mortalidad materna y neonatal. Rev Peru Investig Matern Perinat 2021; 10(3): 7-8

DOI https://doi.org/10.33421/inmp.2021246

Recibido: 22-09-2021 Aceptado: 30-09-2021 
la Morbilidad Materna Extremadamente graves, capacitaciones organizadas para la implementación del uso de la Historia Clínica Perinatal SIP de "Mujeres en Situación de Aborto", ${ }^{\text {, }}$ utilizadas por el CLAP y capacitación en la metodología para la implementación del Modelo de Atención Prenatal basada en el concepto de Pirámide Invertida ${ }^{7,8}$. Es importante que el personal de salud esté permanentemente capacitado en reanimación cardio-respiratoria del adulto básica y avanzada, en reanimación cardio-respiratoria neonatal y del prematuro, y en el manejo de las principales emergencias obstétricas y neonatales.

Finalmente sin ser menos importante, la promoción de las investigaciones en el área materno/neonatal se puede lograr a través del fortalecimiento de las Unidades de Investigación con personal de salud dedicado exclusivamente a la investigación. Es importante promover la existencia de revistas de investigación, como lo tiene el Instituto Nacional Materno Perinatal, la Revista Peruana de Investigación Materno Perinatal (Rev Peru Investig Matern Perinat, ISSN 2663-113X), que es el órgano oficial de difusión científica del Instituto, indizada en Latindex, DOAJ, ROAD y BOAI, y cuyo objetivo es difundir la producción científica de la especialidad materno perinatal/neonatal y de salud sexual y reproductiva, con la finalidad de contribuir a mejorar la situación de la salud materno perinatal del país y de la región ${ }^{9}$.

En conclusión, las instituciones de alta complejidad deben contribuir a la mejora de la salud materna y neonatal, mediante la disminución de los casos de mortalidad materna y neonatal y la recuperación de los casos de morbilidad materna extremadamente grave. Esto se logra a través de una adecuada gestión de la capacidad resolutiva del establecimiento, lo que implica un adecuado gasto presupuestal en infraestructura, equipos, recursos humanos. Potenciando el recurso humano, cerrando la brecha y mejorando sus competencias. La investigación materno perinatal es fundamental para dirigir los escasos recursos a la priorización de los principales complicaciones del embarazo, parto, y puerperio y de los recién nacidos.

Todo esto es posible con una adecuada ejecución presupuestal transparente y pensando en la salud de todas/os los peruanos. La salud de las gestantes y los recién nacidos debe ser una prioridad nacional ya que es un derecho humano básico y fundamental.

\section{REFERENCIAS BIBLIOGRÁFICAS}

1. Ministerio de Salud. Dirección General de Salud de las Personas. Norma Técnica Categorías de Establecimientos del Sector Salud. Perú. Mayo 2005.

2. Ministerio de Salud. Plan Estratégico Instituciona 2019 2024 ampliado del Ministerio de Salud.https://cdn.www. gob.pe/uploads/document/file/1893242/Plan\%20Estrat\%-
C3\%A9gico\%20Institucional\%20PEI\%202019-2024\%20 \%28Parte\%20uno\%29.pdf.

3. Organización Panamericana de la Salud. Recomendaciones de la Organización Mundial de la Salud sobre atención prenatal para una experiencia positiva del embarazo. ISBN 978-92-75-32033-4. 2018.

4. Organización Panamericana de la Salud. Guías para la atención de las principales emergencias obstétricas - 2a ed. ISBN: 978-92-75-32088-4 elSBN: 978-92-75-32089-1. 2019.

5. Organización Panamericana de la Salud. Recomendaciones para establecer un sistema nacional de vigilancia de la morbilidad materna extremadamente grave en América Latina y el Caribe. ISBN: 978-92-75-32390-8 (impreso). ISBN: 978-92-75-32391-5 (pdf). 2021

6. Organización Panamericana de la Salud. Organización Mundial de la Salud. Centro Latinoamericano de Perinatología. Sistema informatico perinatal manual de uso del programa para el análisis y aprovechamiento de la información. Montevideo, Uruguay. 2010.

7. Nicolaides $\mathrm{KH}$. A model for a new pyramid of prenatal care based on the 11 to 13 weeks' assessment. Prenat Diagn 2011;31(1):3-6.

8. Nicolaides $\mathrm{KH}$. Turning the pyramid of prenatal care. Fetal Diagn Ther. 2011;29(3): 183-96.

9. https://investigacionmaternoperinatal.inmp.gob.pe/index. php/rpinmp. 